\title{
Nullstellenverteilung der großen kanonischen Verteilungsfunktion eines Gittergases in der Bethe-Approximation
}

\author{
H. P. NeUmanN \\ Institut für Physikalische Chemie der Universität Frankfurt am Main \\ (Z. Naturforsch. 24 a, 162-169 [1969]; eingegangen am 21. Oktober 1968)
}

\begin{abstract}
Es werden allgemeine Gleichungen zur Berechnung der Verteilung der Nullstellen von der großen kanonischen Verteilungsfunktion $\Xi(y)$ eines Gittergases in der komplexen Ebene der Fugazität $y$ in Bethescher Näherung angegeben, die nach einem graphischen Verfahren gelöst werden können. Für das zweidimensionale, quadratische Gitter und das dreidimensionale, kubische Gitter werden die Bestimmungsgleichungen für die Nullstellenverteilung mit Hilfe des graphischen Verfahrens explizit gelöst. Die Nullstellenverteilung von $\Xi(y)$ des eindimensionalen Gittergases wird in geschlossener Form angegeben.
\end{abstract}

\section{Einleitung}

Die Kondensationspunkte eines realen Gases im Druck-Volumen-Phasendiagramm sind dadurch gekennzeichnet, daß das Volumen an diesen Stellen eine zweideutige Funktion des Druckes wird. In den grundlegenden Arbeiten von YANG und $\operatorname{LEE}^{1}$ wird klar herausgestellt, daß dieses eigentümliche Verhalten der Druck-Volumen-Isothermen eines realen Gases mathematisch durch Singularitäten des Logarithmus der großen kanonischen Verteilungsfunktion $\Xi(y)$ auf der positiven, reellen Achse der Fugazität $y$ hervorgerufen wird.

Diese Singularitäten sind dabei als Häufungspunkte von Nullstellen der großen kanonischen Verteilungsfunktion $\Xi(y)$ in der komplexen $y$-Ebene zu verstehen. Die mathematische Struktur einer solchen Singularität hängt also wesentlich von der Verteilung der Nullstellen in der komplexen $y$-Ebene ab.

Bisher war es nicht möglich, im allgemeinen Fall eines realen Gases genauere Aussagen über diese Nullstellenverteilung $\mathrm{zu}$ machen. Da andererseits eine eingehendere Kenntnis der Nullstellenverteilung für eine exakte mathematische Diskussion der mit der Kondensation verknüpften Singularität und damit für eine befriedigende Beschreibung des Kondensationsphänomens im Rahmen der statistischen Mechanik unerläßlich ist, scheint es wegen der großen mathematischen Schwierigkeiten im allgemeinen Fall eines realen Gases zweckmäßig zu sein, zunächst einmal für einfachere Modellfälle konkrete Aussagen über die Nullstellenverteilung zu machen. In diesem

1 C. N. Yang u. T. D. Lee, Phys. Rev. 87, 404, 410 [1952].

2 P. C. Hemmer u. E. H. Hauge, Phys. Rev. 133, 1010 [1964].

3 P. C. Hemmer, E. H. Hauge u. J. O. Aasen, J. Math. Phys. 7, 35 [1966].
Sinne wurden bisher einige der bekanntesten Gasmodelle behandelt.

Für das einem realen Gas schon recht ähnelnde Gittergasmodell, welchem wohl bisher mit die am wenigsten einschneidenden Voraussetzungen zugrunde liegen und welches die Koexistenzkurve eines realen Gases zumindest qualitativ gut beschreibt, konnten YANG und $\operatorname{LEE}^{1}$ zeigen, daß die Nullstellen stets auf einem Kreis um den Ursprung in der komplexen $y$-Ebene liegen. Über die Verteilung der Nullstellen auf diesem Kreis konnten jedoch allgemein keine Angaben gemacht werden.

Weitere Veröffentlichungen von anderen Autoren befassen sich mit Nullstellenverteilungen von Spezialfällen wie dem van der Waals-Gas ${ }^{2-4}$, eindimensionalen Gasen harter Stäbchen ${ }^{3,5}$, dem Gittergas in der Weißschen Approximation ${ }^{6}$ und noch anderen Modellen ${ }^{7}$.

Aus den Ergebnissen dieser Publikationen zeichnet sich deutlich ab, daß für die Existenz von Nullstellenhäufungspunkten auf der positiven, reellen $y$ Achse, d. h. für die Kondensation, nur der anziehende Anteil eines Wechselwirkungspotentials verantwortlich zu sein scheint. Der abstoßende Anteil eines Wechselwirkungspotentials scheint dagegen eine Belegung eines bestimmten Bereichs der negativen, reellen $y$-Achse mit Nullstellen zu bewirken und nicht unmittelbar für die Kondensation interessant zu sein.

In der vorliegenden Arbeit wird nun das Problem der Nullstellenverteilung der großen kanonischen

4 T. S. Nilsen, Physica 37, 47 [1967].

5 E. H. Hauge u. P. C. Hemmer, Physica 29, 1338 [1963].

${ }^{6}$ S. Katsura, J. Chem. Phys. 22, 1277 [1954].

7 E. Byckling, Phys. Rev. 140, 1165 [1965]. 
Verteilungsfunktion eines Gittergases in der Betheschen Approximation behandelt.

Das Bethesche Modell eines Gittergases ist eine der ersten Näherungsstufen, bei der die Nahordnung berücksichtigt wird und bei welcher besonders in der Umgebung des kritischen Punktes gegenüber dem einfachen Weißschen Modell verbesserte Resultate erzielt werden. Seine Nullstellenverteilung wäre daher vielleicht gerade im Hinblick auf das Verhalten in der Umgebung der kritischen Temperatur von Interesse.

\section{Die Gleichungen für die Nullstellenverteilung von $\Xi(y)$ und ihre graphische Lösung}

Die Zustandsgleichung eines realen Gases kann man mit Hilfe der Funktion $\chi(y)=1 / V \cdot \ln \Xi(y)$ allgemein in folgender Form schreiben:

$$
\beta P=\chi(y), \quad \varrho=\frac{1}{v}=y \chi^{\prime}(y),
$$

wobei $P$ der Druck, $\varrho$ die Dichte und $v$ das Teilchenvolumen sind.

Die Funktionen $\chi(y)$ und besonders $\chi^{\prime}(y)$ sind nicht nur für die Zustandsgleichung (1) wichtig, sondern was hier hauptsächlich interessiert, für die Nullstellenverteilung $g(s)$ der großen kanonischen Verteilungsfunktion.

Wenn die Nullstellen auf Linien in der komplexen $y$-Ebene verteilt sind, was beim Gittergas immer der Fall ist ${ }^{1}$, so springt $\chi^{\prime}(y)$ auf diesen Nullstellenlinien. Umgekehrt gilt: springt $\chi^{\prime}(y)$ auf gewissen Linien, so liegen dort Nullstellen.

Für die Verteilungsfunktion der Nullstellen $g(s)$ ergibt sich dann ${ }^{1-5}$ :

$$
g(s)=\left|\frac{1}{2 \pi i}\left(\chi_{1}^{\prime}(y)-\chi_{2}^{\prime}(y)\right)\right| .
$$

$\chi_{1}^{\prime}(y), \chi_{2}^{\prime}(y)$ sind die beiden Sprungwerte an der Stelle $y$ auf der Nullstellenlinie.

Im Fall der Betheschen Approximation hat die Ableitung $\chi^{\prime}(y)$ von $\chi(y)$ die Gestalt:

$$
\chi^{\prime}(y)=\frac{1}{2 y}\left[1+\operatorname{Tanh}\left[z \delta-\frac{1}{2}(\ln y+2 \beta z \varphi)\right]\right] .
$$

Die Größe $\delta$ bestimmt sich dabei aus der Gleichung:

$$
X=\exp [-\beta \varphi]=\frac{\operatorname{Sinh}\left[(z-2) \delta-\frac{1}{2}(\ln y+2 \beta z \varphi)\right]}{\operatorname{Sinh}\left[z \delta-\frac{1}{2}(\ln y+2 \beta z \varphi)\right]} .
$$

$\varphi$ ist das Wechselwirkungspotential zwischen nächsten Nachbarn und $z$ die Koordinationszahl. $\beta$ ist die übliche Abkürzung $\beta=1 / k T$.

Von der Betheschen Näherung her ist bekannt, daß die Gl. (4) für jedes reelle $y$ mit $\ln y+2 \beta z \varphi \neq 0$ genau eine Lösung $\delta(y)$ besitzt. Da im Bereich dieser Lösungen die rechte Seite von Gl. (4) eine analytische Funktion sowohl von $\delta$ als auch von $y$ ist, ist $\delta(y)$ ebenfalls eine analytische Funktion in dem Gebiet $\langle y\rangle$ mit $\ln y+2 \beta z \varphi \neq 0$. An diesen Punkten auf der reellen $y$-Achse läßt sich die Funktion $\delta(y)$ und wegen (3) auch die Funktionen $\chi^{\prime}(y)$ und $\chi(y)$ in die komplexe $y$-Ebene analytisch fortsetzen.

An den Stellen $y_{0}$ mit $\ln y_{0}+2 \beta z \varphi=0$ und $X<X_{\mathrm{c}}=(z-2) / z$ hat die Gl. (4) dagegen die beiden Lösungen $\delta\left(y_{0}\right)$ und $-\delta\left(y_{0}\right)$. Auf diesen Punkten ist also $\delta(y)$ nicht mehr eindeutig und analytisch.

Hat man nun $\chi(y)$ bzw. $\chi^{\prime}(y)$ in der ganzen komplexen $y$-Ebene bis auf die singulären Punkte (Sprung. linien von $\chi^{\prime}(y)$ ) analytisch fortgesetzt, so kann man auf den Sprunglinien mit Hilfe von (2) die Nullstellenverteilungsfunktion $g(s)$ ausrechnen.

Die analytische Fortsetzung von (4) gestaltet sich nun nach Einführung der komplexen Zahlen $y=r \cdot e^{i \psi}$ und $\delta=\gamma+i \varepsilon$ folgendermaßen:

$$
X=\frac{\operatorname{Sinh}\left[\left((z-2) \gamma-\ln \sqrt{r / X^{2} z}\right)+i\left((z-2) \varepsilon-\frac{1}{2} \psi\right)\right]}{\operatorname{Sinh}\left[\left(z \gamma-\ln \sqrt{r / X^{2} z}\right)+i\left(z \varepsilon-\frac{1}{2} \psi\right)\right]} .
$$

Durch weitere Umformung erhält man:

$$
\begin{aligned}
& X=\left[\operatorname{Sinh}\left[(z-2) \gamma-\ln \sqrt{r / X^{2 z}}\right] \cdot \cos \left[(z-2) \varepsilon-\frac{1}{2} \psi\right]\right. \\
& \left.\quad+i \operatorname{Cosh}\left[(z-2) \gamma-\ln \sqrt{r / X^{2 z}}\right] \cdot \sin \left[(z-2) \varepsilon-\frac{1}{2} \psi\right]\right] / \\
& {\left[\operatorname{Sinh}\left[z \gamma-\ln \sqrt{r / X^{2 z}}\right] \cdot \cos \left[z \varepsilon-\frac{1}{2} \psi\right]+i \operatorname{Cosh}\left[z \gamma-\ln \sqrt{r / X^{2 z}}\right] \cdot \sin \left[z \varepsilon-\frac{1}{2} \psi\right]\right]} \\
& \quad=\left[\left[\operatorname{Sinh}\left[(z-2) \gamma-\ln \sqrt{r / X^{2 z}}\right] \cdot \operatorname{Sinh}\left[z \gamma-\ln \sqrt{r / X^{2 z}}\right] \cdot \cos \left[(z-2) \varepsilon-\frac{1}{2} \psi\right] \cdot \cos \left[z \varepsilon-\frac{1}{2} \psi\right]\right.\right.
\end{aligned}
$$




$$
\begin{aligned}
& \left.+\operatorname{Cosh}\left[(z-2) \gamma-\ln \sqrt{r / X^{2 z}}\right] \cdot \operatorname{Cosh}\left[z \gamma-\ln \sqrt{r / X^{2 z}}\right] \cdot \sin \left[(z-2) \varepsilon-\frac{1}{2} \psi\right] \cdot \sin \left[z \varepsilon-\frac{1}{2} \psi\right]\right] \\
& -i \cdot\left[\operatorname{Sinh}\left[(z-2) \gamma-\ln \sqrt{r / X^{2 z}}\right] \cdot \operatorname{Cosh}\left[z \gamma-\ln \sqrt{r / X^{2 z}}\right] \cdot \cos \left[(z-2) \varepsilon-\frac{1}{2} \psi\right] \cdot \sin \left[z \varepsilon-\frac{1}{2} \psi\right]\right. \\
& -\operatorname{Cosh}\left[(z-2) \gamma-\ln \sqrt{r / X^{2 z}}\right] \cdot \operatorname{Sinh}\left[z \gamma-\ln \sqrt{r / X^{2 z}}\right] \\
& \left.\left.\cdot \sin \left[(z-2) \varepsilon-\frac{1}{2} \psi\right] \cdot \cos \left[z \varepsilon-\frac{1}{2} \psi\right]\right]\right] /\left[\operatorname{Sin}^{2} \mathrm{~h}\left[z \gamma-\ln \sqrt{r / X^{2 z}}\right]\right. \\
& \left.\cdot \cos ^{2}\left[z \varepsilon-\frac{1}{2} \psi\right]+\operatorname{Cos}^{2} \mathrm{~h}\left[z \gamma-\ln \sqrt{r / X^{2 z}}\right] \cdot \sin ^{2}\left[z \varepsilon-\frac{1}{2} \psi\right]\right] .
\end{aligned}
$$

$X$ ist nun eine reelle Zahl. Damit verschwindet oben der Imaginärteil. Man erhält also für das komplexe $\delta=\gamma+i \varepsilon$ als analytische Fortsetzung an Stelle der einen Gl. (4) für das reelle $\delta$ der Betheschen Approximation die beiden Bestimmungsgleichungen:

$$
\begin{aligned}
X= & {\left[\operatorname{Sinh}\left[(z-2) \gamma-\ln \sqrt{r / X^{2 z}}\right] \cdot \operatorname{Sinh}\left[z \gamma-\ln \sqrt{r / X^{2 z}}\right] \cdot \cos \left[(z-2) \varepsilon-\frac{1}{2} \psi\right] \cdot \cos \left[z \varepsilon-\frac{1}{2} \psi\right]\right.} \\
& +\operatorname{Cosh}\left[(z-2) \gamma-\ln \sqrt{r / X^{2 z}}\right] \cdot \operatorname{Cosh}\left[z \gamma-\ln \sqrt{r / X^{2 z}}\right] \\
& \left.\cdot \sin \left[(z-2) \varepsilon-\frac{1}{2} \psi\right] \cdot \sin \left[z \varepsilon-\frac{1}{2} \psi\right]\right] /\left[\operatorname{Sin}^{2} \mathrm{~h}\left[z \gamma-\ln \sqrt{r / X^{2 z}}\right]\right. \\
& \left.\cdot \cos ^{2}\left[z \varepsilon-\frac{1}{2} \psi\right]+\operatorname{Cos}^{2} \mathrm{~h}\left[z \gamma-\ln \sqrt{r / X^{2 z}}\right] \cdot \sin ^{2}\left[z \varepsilon-\frac{1}{2} \psi\right]\right], \\
{[\operatorname{Sinh}[} & \left.(z-2) \gamma-\ln \sqrt{r / X^{2 z}}\right] \cdot \operatorname{Cosh}\left[z \gamma-\ln \sqrt{r / X^{2 z}}\right] \cdot \cos \left[(z-2) \varepsilon-\frac{1}{2} \psi\right] \cdot \sin \left[z \varepsilon-\frac{1}{2} \psi\right] \\
& -\operatorname{Cosh}\left[(z-2) \gamma-\ln \sqrt{r / X^{2 z}}\right] \cdot \operatorname{Sinh}\left[z \gamma-\ln \sqrt{r / X^{2 z}}\right] \\
& \left.\cdot \sin \left[(z-2) \varepsilon-\frac{1}{2} \psi\right] \cdot \cos \left[z \varepsilon-\frac{1}{2} \psi\right]\right] /\left[\operatorname{Sin}^{2} \mathrm{~h}\left[z \gamma-\ln \sqrt{r / X^{2 z}}\right]\right. \\
& \left.\cdot \cos ^{2}\left[z \varepsilon-\frac{1}{2} \psi\right]+\operatorname{Cos}^{2} \mathrm{~h}\left[z \gamma-\ln \sqrt{r / X^{2 z}}\right] \cdot \sin ^{2}\left[z \varepsilon-\frac{1}{2} \psi\right]\right]=0 .
\end{aligned}
$$

Die analytische Fortsetzung von (3) in die komplexe $y$-Ebene ist einfach:

$$
\chi^{\prime}(y)=\frac{1}{2 \cdot X^{2 z+i \psi}} \cdot\left[1+\operatorname{Tanh}\left[\left(z \gamma-\frac{1}{2}(\ln y+2 \beta z \varphi)\right)+i\left(z \varepsilon-\frac{1}{2} \psi\right)\right]\right] .
$$

Nullstellen von $\Xi(y)$ treten nun in der komplexen $y$-Ebene nur dort auf, wo $\chi^{\prime}(y)$. Sprungstellen hat. Sprungstellen von $\chi^{\prime}(y)$ können aber nur die singulären Punkte des Gleichungssystems (6), (7) sein, bei welchen zu einer bestimmten Zahl $y$ zwei Lösungen $\delta_{1}$ und $\delta_{2}$ existieren. Wo diese Stellen prinzipiell liegen, kann man aus (6), (7) und (8) ablesen.

Hat man zwei konjugiert komplexe Zahlen $y$ und $\bar{y}$, so folgt: $\delta(\bar{y})=\bar{\delta}(y)$ und weiter $\chi^{\prime}(\bar{y})=\bar{\chi}^{\prime}(y)$, da wegen (6) und (7) aus $\psi \rightarrow-\psi \varepsilon \rightarrow-\varepsilon$ folgt. Die Nullstellen sind also symmetrisch zur reellen $y$-Achse verteilt ${ }^{1}$.

Die Funktion $\varepsilon(r, \psi)$ ist mehrdeutig, denn mit $\tilde{\varepsilon}(r, \psi)$ aus dem Intervall $0 \leqq|\tilde{\varepsilon}| \leqq \pi$, wobei $\psi$ im Intervall $0 \leqq|\psi| \leqq \pi$ liegt, sind auch $\tilde{\varepsilon} \pm n \pi$ ( $n$ ist eine natürliche Zahl) Lösungen von (6) und (7). Allerdings sind dies keine wesentlich neuen Lösungen, da wegen (8) $\chi^{\prime}(y)$ und damit wegen $(2)$ auch $g(s)$ invariant unter der Transformation $\varepsilon \rightarrow \varepsilon \pm n \pi$ bleiben. Aus der später durchgeführten graphischen Konstruktion wird dann ersichtlich, daß $\tilde{\varepsilon}(r, \psi)$ mit $|\psi|$ aus $\langle 0, \pi\rangle$ eine eindeutige Funktion von $\psi$ ist.

Da $\varepsilon$ nun als eindeutig angenommen werden kann, ist eine Zweideutigkeit der Lösung nur noch für $\gamma$ möglich.

Ist $(\gamma, \varepsilon)$ nun eine Lösung von (6) und (7) zu gegebenem $\psi$ und $\ln \sqrt{r_{1} / X^{2 z}}$, so existiert auch eine weitere Lösung $(-\gamma, \varepsilon)$ mit $\ln \sqrt{r_{2} / X^{2 z}}=-\ln \sqrt{r_{1} / X^{2 z}}$. Bei der anschließend zu behandelnden graphischen Methode wird wieder ersichtlich, daß für $\ln V r / X^{2 z} \neq 0 \quad \gamma$ stets eine eindeutige Funktion $\gamma(r, \psi)$ ist.

Die Nullstellen können also in Übereinstimmung mit der allgemeinen Theorie von YANG und LEE ${ }^{1}$ nur auf dem Kreis mit dem Radius $r_{0}=\exp [-2 \beta z \varphi]=X^{2 z}$ liegen, wo $\ln \sqrt{r / X^{2 z}}$ verschwindet, denn nur dort können für gewisse Werte von $X$ und $\psi$ zwei von Null verschiedene Lösungen $\gamma$ und $-\gamma$ bzw. $\delta_{1}=\gamma+i \varepsilon$ und $\delta_{2}=-\gamma+i \varepsilon$ nebeneinander existieren. Mit Ausnahme dieser singulären Stellen ist $\chi(y)$ analytisch in die komplexe $y$-Ebene fortsetzbar.

Die Nullstellenverteilung auf dem Kreis mit dem Radius $r_{0}$ kann man nun wegen (2) und (8) folgendermaßen ausrechnen:

$$
\begin{aligned}
g(s) & =\frac{1}{4 \pi X^{2 z}+i \psi \cdot i}\left[\operatorname{Tanh}\left[z \gamma+i\left(z \varepsilon-\frac{1}{2} \psi\right)\right]-\operatorname{Tanh}\left[-z \gamma+i\left(z \varepsilon-\frac{1}{2} \psi\right)\right]\right]=g(\psi) \\
& =\frac{1}{4 \pi X^{2 z}} \mid \frac{\operatorname{Sinh} z \gamma \cdot \operatorname{Cosh} z \gamma+i \cdot \sin \left(z \varepsilon-\frac{1}{2} \psi\right) \cdot \cos \left(z \varepsilon-\frac{1}{2} \psi\right)}{\operatorname{Cos}^{2} \mathrm{~h} \cdot \cos ^{2}\left(z \varepsilon-\frac{1}{2} \psi\right)+\operatorname{Sin} 2 \mathrm{~h} z \gamma \cdot \sin ^{2}\left(z \varepsilon-\frac{1}{2} \psi\right)}+\frac{\operatorname{Sinh} z \gamma \cdot \operatorname{Cosh} z \gamma-i \sin \left(z \varepsilon-\frac{1}{2} \psi\right) \cdot \cos \left(z \varepsilon-\frac{1}{2} \psi\right)}{\operatorname{Cos}^{2} z \gamma \cdot \cos ^{2}\left(z \varepsilon-\frac{1}{2} \psi\right)+\operatorname{Sin}^{2} \mathrm{~h} z \gamma \cdot \sin ^{2}\left(z \varepsilon-\frac{1}{2} \psi\right)} .
\end{aligned}
$$


Daraus folgt für $g(\psi)$ :

$$
g(\psi)=\frac{X^{-2} z}{2 \pi} \cdot \frac{\operatorname{Sinh} z \gamma \cdot \operatorname{Cosh} z \gamma}{\operatorname{Cos}^{2} \mathrm{~h} z \gamma \cdot \cos ^{2}\left(z \varepsilon-\frac{1}{2} \psi\right)+\operatorname{Sin}^{2} \mathrm{~h} z \gamma \cdot \sin ^{2}\left(z \varepsilon-\frac{1}{2} \psi\right)} .
$$

Für die Auswertung der Gln. (6), (7) und (9) auf graphischem Wege ist es angebracht, sie zuerst in eine dem graphischen Verfahren besser angepaßte Form zu bringen.

Auf dem Nullstellenkreis selbst interessiert nur der Bereich, in dem Nullstellen vorhanden sind. Dort muß aber nach den oben angestellten Überlegungen $\gamma$ von Null verschieden sein. Damit sind die Nenner in (6), (7) und (9) ebenfalls von Null verschieden.

Aus (7) folgt ferner, daß in diesem Falle weder $\cos \left[(z-2) \varepsilon-\frac{1}{2} \psi\right]$ noch $\cos \left[z \varepsilon-\frac{1}{2} \psi\right]$ Null sein können. Man kann also in (6), (7) und (9) in geeigneter Weise mit dem Faktor Cosh $(z-2) \gamma \cdot \operatorname{Cosh} z \gamma$ $\cdot \cos \left[(z-2) \varepsilon-\frac{1}{2} \psi\right] \cdot \cos \left(z \varepsilon-\frac{1}{2} \psi\right)$ kürzen. Verwendet man noch die Formel $\sqrt{1+\operatorname{tg}^{2} \varepsilon}=1 / \cos \varepsilon$, so ändern sich (6), (7) und (9) in folgende Beziehungen ab:

$$
\begin{gathered}
X=\Phi(\gamma, \psi)=\sqrt{\frac{1+\operatorname{tg}^{2}\left[z \varepsilon-\frac{1}{2} \psi\right]}{1+\operatorname{tg}^{2}\left[(z-2) \varepsilon-\frac{1}{2} \psi\right]}} \cdot \frac{\operatorname{Cosh}(z-2) \gamma}{\operatorname{Cosh} z \gamma} \\
\cdot \frac{\left[\operatorname{Tanh}(z-2) \gamma \cdot \operatorname{Tanh} z \gamma+\operatorname{tg}\left[(z-2) \varepsilon-\frac{1}{2} \psi\right] \cdot \operatorname{tg}\left[z \varepsilon-\frac{1}{2} \psi\right]\right]}{\left[\operatorname{Tan}^{2} z \gamma+\operatorname{tg}^{2}\left[z \varepsilon-\frac{1}{2} \psi\right]\right]}, \\
\operatorname{tg}\left[z \varepsilon-\frac{1}{2} \psi\right]=\frac{\operatorname{Tanh} z \gamma}{\operatorname{Tanh}(z-2) \gamma} \cdot \operatorname{tg}\left[(z-2) \varepsilon-\frac{1}{2} \psi\right] \\
g(\psi)=\frac{X-2 z}{2 \pi} \cdot \frac{\operatorname{Tanh} z \gamma \cdot\left[1+\operatorname{tg}^{2}\left[z \varepsilon-\frac{1}{2} \psi\right]\right]}{\left[1+\operatorname{Tan}^{2} h \gamma \cdot \operatorname{tg}^{2}\left[z \varepsilon-\frac{1}{2} \psi\right]\right]}
\end{gathered}
$$

Die Gl. (11) läßt sich nun, wie aus Abb. 1 hervorgeht, graphisch lösen.

$\mathrm{Zu}$ diesem Zweck wählt man für die Werte des Parameters $\gamma$ eine Gruppe von Wertepaaren

$$
\operatorname{tg}\left[(z-2) \varepsilon-\frac{1}{2} \psi\right] \text { und } \operatorname{tg}\left[z \varepsilon-\frac{1}{2} \psi\right]
$$

aus, die (11) erfüllen. Aus Abb. 1 kann man dann unmittelbar zu jedem Parameterwert $\gamma$ eine Gruppe von zugeordneten Wertepaaren $2 \varepsilon$ und $\frac{1}{2} \psi$ ablesen. Mit Hilfe dieser Wertepaare kann man sofort die Kurvenscharen:

$2 \varepsilon=2 \varepsilon_{\gamma}\left(\frac{1}{2} \psi\right), \quad \operatorname{tg}\left[(z-2) \varepsilon-\frac{1}{2} \psi\right]=\operatorname{tg}\left[(z-2) \varepsilon-\frac{1}{2} \psi\right]_{\gamma}\left(\frac{1}{2} \psi\right) \quad$ und $\operatorname{tg}\left[z \varepsilon-\frac{1}{2} \psi\right]=\operatorname{tg}\left[z \varepsilon-\frac{1}{2} \psi\right]_{\gamma}\left(\frac{1}{2} \psi\right)$ mit dem Scharparameter $\gamma$ aufzeichnen. Die so ermittelten Werte von $\operatorname{tg}\left[(z-2) \varepsilon-\frac{1}{2} \psi\right]$ und $\operatorname{tg}\left[z \varepsilon-\frac{1}{2} \psi\right]$, die, wie aus Abb. 1 hervorgeht, negativ für positive Werte von $\psi$ sind, kann man in Gl. (10) einsetzen. Man erhält dann die Kurvenschar $\Phi(\gamma, \psi)$ mit dem Scharparameter $\psi$, die in Abb. 2 dargestellt ist. Diese Kurvenschar kann mit jeder beliebigen Geraden $\Phi=X=$ const mit $0 \leqq X \leqq 1$ zum Schnitt gebracht werden.

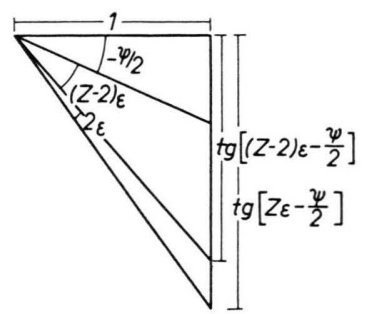

Abb. 1. Figur zur Konstruktion der graphischen Lösung von Gl. (11) für beliebige $\gamma$-Werte.

Abb. 2. Konstruktionsfigur zur graphischen Lösung von Gl. (10).

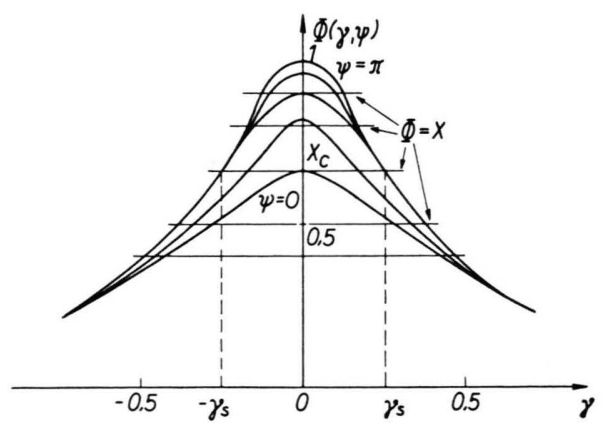

Die Schnittpunkte haben Abszissen $\gamma_{\mathrm{s}}=\gamma_{\mathrm{s}}\left(X, \frac{1}{2} \psi\right)$. Man braucht sich aus den beiden mit Hilfe von Abb. 1 konstruierten Scharen $\operatorname{tg}\left[(z-2) \varepsilon-\frac{1}{2} \psi\right]$ und $\operatorname{tg}\left[z \varepsilon-\frac{1}{2} \psi\right]$ nur noch die Kurve $\operatorname{tg}\left[z \varepsilon-\frac{1}{2} \psi\right]$ mit 
dem Scharparameter $\gamma_{\mathrm{s}}\left(X, \frac{1}{2} \psi\right)$ zu wählen und deren Werte sowie die Abschnitte $\gamma_{\mathrm{s}}\left(X, \frac{1}{2} \psi\right)$ in Gl. (12) einzusetzen, um die Schar $g_{x}(\psi)$ der Nullstellenverteilungen mit dem Scharparameter $X$ längs des Kreises mit dem Radius $r_{0}$ auszurechnen.

Das eben geschilderte Verfahren zur Lösung von (10) und (11) läßt sich auch auf den Fall $\ln \sqrt{r / X^{2 \bar{z}}} \neq 0$ ausdehnen. Man erhält dann statt einer Kurvenschar über der Ebene mit den Koordinaten $\left\langle\ln \sqrt{r / X^{2} z}, \gamma\right\rangle$ eine Flächenschar $\Phi_{\psi}(r, \gamma)$ mit $\psi$ als Scharparameter ähnlich wie in Abb. 2, die mit den Flächen $\Phi=X$ geschnitten wird. Damit ergeben sich eindeutige Lösungen $\gamma(r, \psi)$ und $\varepsilon(r, \psi)$ und weiter reguläre Lösungen $\delta(y)$. Mit Hilfe von (8) erhält man endlich die analytischen Fortsetzungen $\chi(y)$.

Bei den mittels der Gln. (10) bis (12) errechneten Nullstellenverteilungen $g(\psi)$ interessiert unter anderem das Ende der Nullstellenverteilung, kurz auch „Kopf“ genannt. An dieser Stelle muß $g(\psi)$, d. h. $\gamma$ verschwinden. Die Beziehungen (10) und (11) vereinfachen sich dann zu:

$$
\begin{gathered}
X=\Phi\left(0, \psi_{\mathrm{K}}\right)=\sqrt{\frac{1+\operatorname{tg}^{2}\left[(z-2) \varepsilon-\frac{1}{2} \psi_{\mathrm{K}}\right]}{1+\operatorname{tg}^{2}\left[z \varepsilon-\frac{1}{2} \psi_{\mathrm{K}}\right]}} \cdot \frac{\operatorname{tg}\left[(z-2) \varepsilon-\frac{1}{2} \psi_{\mathrm{K}}\right]}{\operatorname{tg}\left[z \varepsilon-\frac{1}{2} \psi_{\mathrm{K}}\right]}, \\
\operatorname{tg}\left[z \varepsilon-\frac{1}{2} \psi_{\mathrm{K}}\right]=\frac{z}{z-2} \cdot \operatorname{tg}\left[(z-2) \varepsilon-\frac{1}{2} \psi_{\mathrm{K}}\right] .
\end{gathered}
$$

Aus diesen Gleichungen kann man die Winkelkoordinate $\psi_{\mathrm{K}}$ des „Kopfes“ als Funktion von $X$ ausrechnen. Graphisch ist $\psi_{\mathrm{K}}$ nach Abb. 2 als derjenige Scharparameter bestimmt, dessen zugeordnete Kurve nur noch im Scheitelpunkt durch die Gerade $X=\Phi$ berührt wird.

Wenn der „Kopf“ der Nullstellenverteilung zur reellen, positiven $y$-Achse aufschließt, d. h. $\psi_{\mathrm{K}}$ gegen Null strebt, folgt aus (13) und (14) für $X$ die bekannte Formel für den kritischen Wert $X_{\mathrm{c}}$ aus der Betheschen Approximation:

$$
X_{\mathrm{c}}=(z-2) / z
$$

Dieses Ergebnis steht ebenfalls im Einklang mit der Theorie von YANG und LEE ${ }^{1}$.

Aussagen analytischer Form über die Nullstellenverteilung $g(\psi)$ kann man nur näherungsweise in einigen Spezialfällen machen:

\section{a) Für sehr tiefe Temperaturen $(X \approx 0)$}

Aus Abb. 2 wird klar, daß für kleine $X \gamma_{s}$ sehr groß wird. Für große $\gamma_{s}$ gilt aber wegen Gl. (11) $\operatorname{tg}\left[(z-2) \varepsilon-\frac{1}{2} \psi\right] \cong \operatorname{tg}\left[z \varepsilon-\frac{1}{2} \psi\right] \cong-\operatorname{tg}\left[\frac{1}{2} \psi\right]$. Außerdem ist Tanh $z \gamma \approx 1$. Die Formel (12) vereinfacht sich dann zu:

$$
g(\psi)=\frac{X^{-2 z}}{2 \pi} .
$$

Die Nullstellen sind also homogen auf dem Kreis mit dem immer kleiner werdenden Radius $r_{0}=X^{2 z}$ verteilt. Die Verteilungsdichte $g(\psi)$ wächst für kleiner werdendes $X$ nach Unendlich.

\section{b) Für sehr hohe Temperaturen $(X \approx 1)$}

Aus Abb. 2 geht hervor, daß für $X \approx 1 \gamma_{\mathrm{s}}$ sehr klein ist. Die Gln. (10) und (11) können dann zunächst einmal grob durch (13) und (14) ersetzt werden. $X \approx 1$ ist aber nur dann mit (13) und (14) verträglich, wenn $\psi \approx \pi$ ist.

Da dann sowohl $\operatorname{tg}\left[(z-2) \varepsilon-\frac{1}{2} \psi\right]$ als auch $\operatorname{tg}\left[z \varepsilon-\frac{1}{2} \psi\right]$ negativ unendlich ist, ist es zweckmäßig, in den Gln. (10), (11) und (12) von den Tangensfunktionen zu den entsprechenden Kotangensfunktionen überzugehen, diese an der Stelle $\pi$ in Taylor-Reihen zu entwickeln und nach dem ersten Glied abzubrechen.

Gleichzeitig können, da $\gamma_{\mathrm{s}} \ll 1$ ist, die hyperbolischen Funktionen noch nach $\gamma$ entwickelt und durch das lineare, ggf. quadratische Glied approximiert werden. Aus Gl. (11) bzw. (14) entsteht dann die einfache Gleichung

$$
\operatorname{ctg}\left[z \varepsilon-\frac{1}{2} \psi\right]=\frac{z-2}{z} \operatorname{ctg}\left[(z-2) \varepsilon-\frac{1}{2} \psi\right] \approx\left[z \mathrm{~d} \varepsilon-\mathrm{d}\left(\frac{1}{2} \psi\right)\right]=\frac{z-2}{z}\left[(z-2) \mathrm{d} \varepsilon-\mathrm{d}\left(\frac{1}{2} \psi\right)\right],
$$


mit der Lösung

$$
\mathrm{d} \varepsilon=\frac{1}{2(1-z)} \mathrm{d}\left(\frac{1}{2} \psi\right) .
$$

Damit kann man näherungsweise setzen:

$$
\operatorname{ctg}\left[(z-2) \varepsilon-\frac{1}{2} \psi\right] \approx \frac{3 z-4}{2(1-z)} \mathrm{d}\left(\frac{1}{2} \psi\right), \quad \operatorname{ctg}\left[z \varepsilon-\frac{1}{2} \psi\right] \approx \frac{3 z-2}{2(1-z)} \mathrm{d}\left(\frac{1}{2} \psi\right) .
$$

Die Gl. (10) geht unter Verwendung obiger Resultate wegen $\gamma \ll 1$ in eine einfache quadratische Gleichung für $\gamma$ über:

$$
X=\frac{\left(1+\frac{1}{2} \operatorname{ctg}\left[z \varepsilon-\frac{1}{2} \psi\right]\right) \cdot\left(1-(z-2)^{2} \gamma^{2}\right)}{\left(1+\frac{1}{2} \operatorname{ctg}\left[(z-2) \varepsilon-\frac{1}{2} \psi\right]\right) \cdot\left(1-z^{2} \gamma^{2}\right)} \approx \frac{\left(1+\frac{3 z-2}{4(1-z)} \mathrm{d}\left(\frac{1}{2} \psi\right)\right) \cdot\left(1-(z-2)^{2} \gamma^{2}\right)}{\left(1+\frac{3 z-4}{4(1-z)} \mathrm{d}\left(\frac{1}{2} \psi\right)\right) \cdot\left(1-z^{2} \gamma^{2}\right)} .
$$

An Stelle von (12) erhält man näherungsweise:

$$
g(\psi) \approx \frac{X^{-2 z}}{2 \pi} \frac{z \gamma \cdot\left[1+\operatorname{ctg}^{2}\left[z \varepsilon-\frac{1}{2} \psi\right]\right]}{\left[(z \gamma)^{2}+\operatorname{ctg}^{2}\left[z \varepsilon-\frac{1}{2} \psi\right]\right]} \approx \frac{X^{-2 z}}{2 \pi} \cdot \frac{z \gamma \cdot\left[1+\frac{(3 z-2)^{2}}{4(1-z)^{2}} \mathrm{~d}^{2}\left(\frac{1}{2} \psi\right)\right]}{\left[(z \gamma)^{2}+\frac{3 z-2}{4(1-z)^{2}} \mathrm{~d}^{2}\left(\frac{1}{2} \psi\right)\right]} .
$$

Setzt man darin die Lösung aus der obigen quadratischen Gleichung:

$$
\gamma=\sqrt{\frac{(1-X)+\frac{1}{4(1-z)}((3 z-2)-(3 z-4) X) \mathrm{d}\left(\frac{1}{2} \psi\right)}{\left(z^{2} X-(z-2)^{2}\right)+\frac{1}{4(1-z)}\left((3 z-4) z^{2} X-(3 z-2)(z-2)^{2}\right) \mathrm{d}\left(\frac{1}{2} \psi\right)}}
$$

ein, so gewinnt man schließlich die Nullstellenverteilung $g(\psi)$ für hohe Temperaturen:

$$
\begin{aligned}
& g(\psi)=\frac{X^{-2} z}{2 \pi} \cdot z \cdot \sqrt{\frac{\left[(1-X)+\frac{1}{4(1-z)}[(3 z-2)-(3 z-4) X] \mathrm{d}\left(\frac{1}{2} \psi\right)\right]}{\left.\left[z^{2} X-(z-2)^{2}\right]+\frac{1}{4(1-z)} \cdot\left[(3 z-4) z^{2} X-(3 z-2)(z-2)^{2}\right] \mathrm{d}\left(\frac{1}{2} \psi\right)\right]}} \\
& \cdot\left[\left(1+\frac{(3 z-2)^{2}}{4(1-z)^{2}} \mathrm{~d}^{2}\left(\frac{1}{2} \psi\right)\right) \cdot\left(\left(z^{2} \cdot X-(z-2)^{2}\right)+\frac{1}{4(1-z)} \cdot\left((3 z-4) z^{2} \cdot X-(3 z-2)(z-2)^{2}\right) \mathrm{d}\left(\frac{1}{2} \psi\right)\right)\right] / \\
& {\left[z ^ { 2 } \cdot \left((1-X)+\frac{1}{4(1-z)} \cdot((3 z-2)-(3 z-4) X) \mathrm{d}\left(\frac{1}{2} \psi\right)+\frac{(3 z-2)^{2}}{4(1-z)^{2}} \mathrm{~d}^{2}\left(\frac{1}{2} \psi\right)\right.\right.} \\
&\left.\cdot\left(\left(z^{2} X-(z-2)^{2}\right)+\frac{1}{4(1-z)} \cdot\left((3 z-4) \cdot z^{2} X-(3 z-2)(z-2)^{2}\right) d\left(\frac{1}{2} \psi\right)\right)\right]
\end{aligned}
$$

Die Winkelkoordinate $\psi_{\mathrm{K}}$ des Kopfes ist dadurch definiert, daß $\gamma$ und $g(\psi)$ den Wert Null annehmen. Wegen (17) muß $\psi_{\mathrm{K}}$ daher der Gleichung

$$
(1-X)+\frac{1}{4(1-z)}[(3 z-2)-(3 z-4) X] \cdot \frac{1}{2} \psi_{\mathrm{K}}
$$

genügen.

Daraus folgt für $\psi_{\mathrm{K}}$ :

$$
\psi_{\mathrm{K}}=\frac{8 \cdot(X-1)(1-z)}{[(3 z-2)-(3 z-4) X]} .
$$

Mit Ausnahme der eben behandelten beiden Spezialfälle a) und b) wird man fast immer auf Näherungsverfahren, wie zum Beispiel der in diesem Abschnitt beschriebenen graphischen Methode, zurückgreifen müssen, um die Nullstellenverteilung $g(\psi)$ quantitativ zu berechnen.

Das graphische Verfahren versagt beim eindimensionalen Gittergas. Dieser Fall wird daher gesondert im nächsten Abschnitt behandelt. Anwendungen auf das zweidimensionale, quadratische Gitter und das dreidimensionale, kubische Gitter werden im übernächsten Abschnitt gebracht. 


\section{Die Nullstellenverteilung $g(\psi)$ für das eindimensionale Gittergasmodell}

Im Fall des eindimensionalen Betheschen Gittergases mit der Koordinationszahl $z=2$ sind die Umformungen, die von den Ausgangsgleichungen (6), (7) und (9) auf die dem graphischen Verfahren zugrunde liegenden Gln. (10) bis (12) führen, nicht mehr möglich. Die Gln. (6), (7) und (9) vereinfachen sich auf der anderen Seite derart, daß geschlossene, konkrete Aussagen über $g(\psi)$ möglich werden. An Stelle von (6), (7) und (9) erhält man auf dem Kreis der Nullstellen ( $\ln \sqrt{r} / X^{2 z}=0$ ) die Gleichungen

$$
\begin{gathered}
X=\Phi(\gamma, \psi)=-\frac{\operatorname{Cosh} 2 \gamma \cdot \sin \frac{1}{2} \psi \cdot \operatorname{Sin}\left(2 \varepsilon-\frac{1}{2} \psi\right)}{\operatorname{Sin}^{2} \mathrm{~h} 2 \gamma \cdot \cos ^{2}\left(2 \varepsilon-\frac{1}{2} \psi\right)+\operatorname{Cos}^{2} \mathrm{~h} 2 \gamma \cdot \sin ^{2}\left(2 \varepsilon-\frac{1}{2} \psi\right)}, \\
\frac{\operatorname{Sinh} 2 \gamma \cdot \sin \frac{1}{2} \psi \cdot \cos \left(2 \varepsilon-\frac{1}{2} \psi\right)}{\operatorname{Sin}^{2} \mathrm{~h} 2 \gamma \cdot \cos ^{2}\left(2 \varepsilon-\frac{1}{2} \psi\right)+\operatorname{Cos}^{2} \mathrm{~h} 2 \gamma \cdot \sin ^{2}\left(2 \varepsilon-\frac{1}{2} \psi\right)}=0, \\
g(\psi)=\frac{X^{-4}}{2 \pi} \cdot \frac{\operatorname{Sinh} 2 \gamma \cdot \operatorname{Cosh} 2 \gamma}{\operatorname{Cos}^{2} \mathrm{~h} 2 \gamma \cdot \cos ^{2}\left(2 \varepsilon-\frac{1}{2} \psi\right)+\operatorname{Sin}^{2} \mathrm{~h} 2 \gamma \cdot \sin ^{2}\left(2 \varepsilon-\frac{1}{2} \psi\right)} .
\end{gathered}
$$

Die Gl. (20) ist auf dem Nullstellenkreis auf den Punkten, welche Nullstellen sind und wo folglich auch $\gamma \neq 0$ ist, außer für $\psi=0$ nur zu befriedigen, wenn $\cos \left(2 \varepsilon-\frac{1}{2} \psi\right)=0$ gilt. Daraus folgt unter Berücksichtigung von (8) wegen $X>0$ :

$$
\tilde{\varepsilon}=-\frac{1}{4}\left(\pi-\frac{1}{2} \psi\right) \text {. }
$$

Diese Lösung $\tilde{\varepsilon}$ ist natürlich ebenso wie die Lösung $\tilde{\varepsilon}$ des im vorigen Abschnitt behandelten, allgemeinen Problems nicht eindeutig. Mit $\tilde{\varepsilon}$ sind auch $\varepsilon=\tilde{\varepsilon} \pm n \pi$ mit der natürlichen Zahl $n$ wieder Lösungen der Gln. (19) bis (21), die allerdings zu denselben Ergebnissen führen und folglich nicht weiter für die Nullstellenverteilung interessieren.

Auf den Punkten des Nullstellenkreises, die keine Nullstellen sind, muß $\gamma=0$ und $g(\psi)=0$ gelten. Dies ist aber nur mit Gl. (21) verträglich, wenn $\cos \left(2 \varepsilon-\frac{1}{2} \psi\right)$ von Null verschieden ist, d. h. die Beziehung (22) verletzt wird.

Man erhält dann statt der beiden Gln. (19) und (20) nur eine sehr vereinfachte Form der Gl. (19), aus der man leicht die Lösung $\varepsilon=\varepsilon(X, \psi)$ finden kann.

Wegen (22) vereinfachen sich (19) und (21) beträchtlich. Es resultiert:

$$
\begin{array}{r}
X=\Phi(\gamma, \psi)=\frac{\sin \frac{1}{2} \psi}{\operatorname{Cosh} 2 \gamma}, \\
g(\psi)=\frac{X^{-4}}{2 \pi} \cdot \frac{\operatorname{Cos}^{2} \mathrm{~h} 2 \gamma}{\sqrt{\operatorname{Cos}^{2} \mathrm{~h} 2 \gamma-1}} .
\end{array}
$$

Die durch Gl. (23) definierte Kurvenschar $\Phi(\gamma, \psi)$ zeigt einen ähnlichen Verlauf wie die in Abb. 2 aufgezeichnete Schar mit dem Unterschied, daß die Kurve mit dem Parameter $\psi=0$ einfach durch die Gerade $\Phi=0$ dargestellt wird. Die beiden „Köpfe“ der Nullstellenverteilung $g(\psi)$ nähern sich also nur für ganz tiefe Temperaturen der reellen, positiven $y$ Achse, erreichen diese aber nie für endliche Temperaturen. Es gibt also keinen kritischen Punkt.

Setzt man in (23) $\gamma=0$, so erhält man die folgende, einfache Gleichung für die Winkelkoordinate $\psi_{\mathrm{K}}$ des „Kopfes" der Nullstellenverteilung:

$$
X=\sin \left(\frac{1}{2} \psi_{\mathrm{K}}\right) .
$$

Für $X<\sin \left(\frac{1}{2} \psi\right)$ kann man die beiden Gln. (23) und (24) kombinieren zur endgültigen Formel für $g(\psi)$ :

$$
g(\psi)=\frac{X^{-4}}{2 \pi} \cdot \frac{\sin \left(\frac{1}{2} \psi\right)}{V \sin ^{2}\left(\frac{1}{2} \psi\right)-X^{2}} .
$$

Nach Formel (26) errechnete Nullstellenverteilungskurven sind in Abb. 3 aufgetragen.

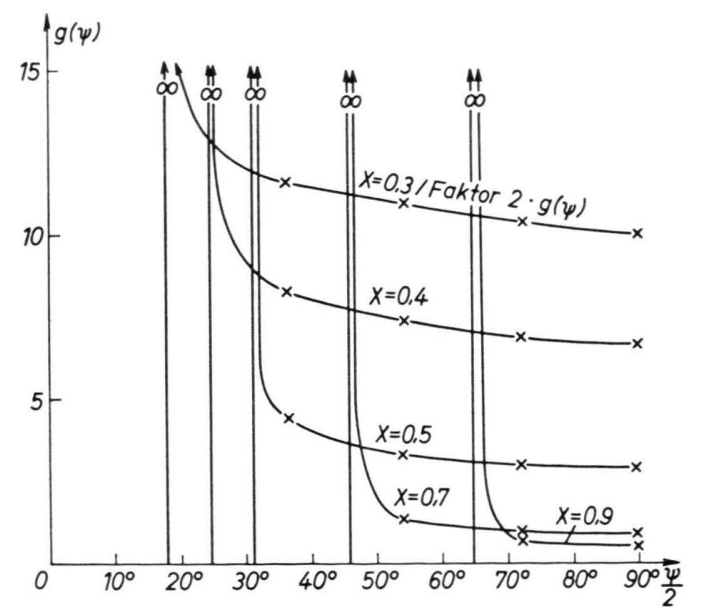

Abb. 3. Nullstellenverteilungskurven für ein eindimensionales Gittergas in Bethescher Näherung mit dem Parameter $X$ bei teilweise verzerrtem Ordinatenmaßstab.

Aus Abb. 3 kann man ersehen, daß die Verteilungskurven $g(\psi)$ an der Stelle des „Kopfes“ Unendlichkeitsstellen haben. 
4. Die Nullstellenverteilungen $g(\psi)$ für das zweidimensionale, quadratische Gittergasmodell und das dreidimensionale, kubische Gittergasmodell auf der Basis der Formeln (10) - (12)

Das im zweiten Abschnitt dargelegte Verfahren kann man direkt zur Berechnung der Nullstellenverteilung für ein Gittergas auf einem quadratischen und kubischen Gitter verwenden. Man braucht dazu in den Beziehungen (10) - (12) nur die Koordinationszahlen $z=4$ bzw. $z=6$ einzusetzen.

Die numerischen Ergebnisse sind in Abb. 4 und 5 aufgezeichnet.

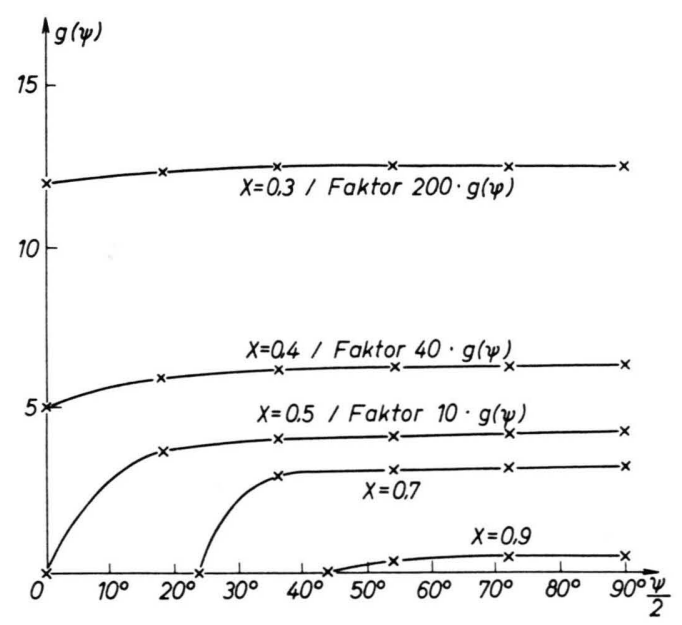

Abb. 4. Nullstellenverteilung für das zweidimensionale Gittergas in Bethescher Näherung mit dem Parameter $X$ bei teilweise geändertem Maßstab der Ordinate.

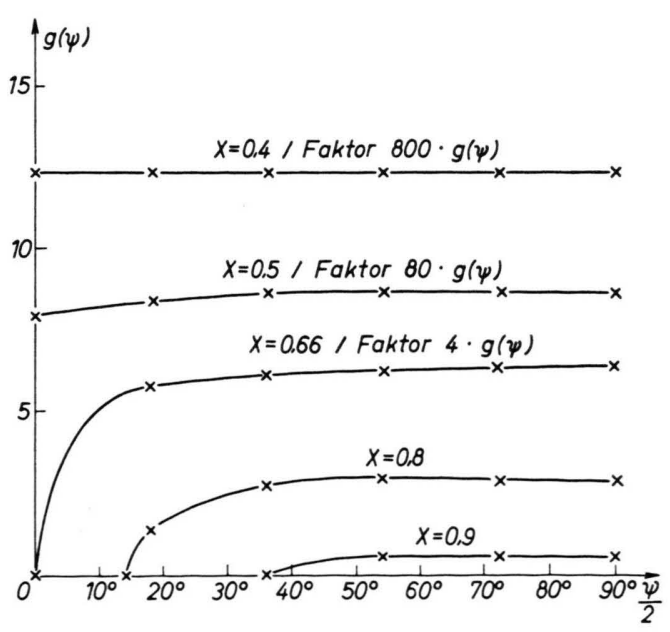

Abb. 5. Nullstellenverteilungskurven mit dem Scharparameter $X$ für das dreidimensionale Gittergas in Bethescher Näherung mit zum Teil variiertem Ordinatenmaßstab.
In Abb. 6 sind vom ein-, zwei- und dreidimensionalen Gittergas die nach den Gln. (13), (14) und (25) errechneten Winkelkoordinaten $\psi_{\mathrm{K}}$ der „Köpfe“ der Nullstellenverteilungen als Funktionen von $X$ aufgetragen.

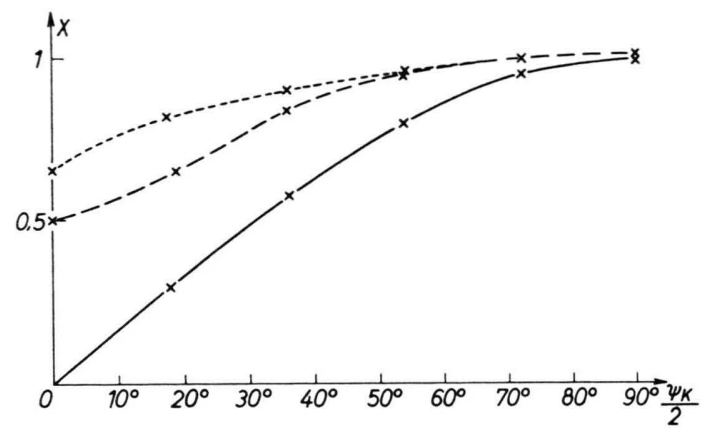

Abb. 6. Die Winkelkoordinaten $\psi_{\mathrm{K}}$ der Köpfe der Nullstellenverteilungen als Funktionen von $X$. Die durchgehende Kurve gehört zum eindimensionalen Gas, die gestrichelte Kurve zum zweidimensionalen Gas und die punktierte Kurve zum dreidimensionalen Gas.

Die Nullstellenverteilunskurven des zwei- und dreidimensionalen Gases zeigen, wie aus Abb. 4 und Abb. 5 hervorgeht, wenig qualitative Unterschiede auf.

Die Nullstellenverteilungskurven des eindimensionalen Gases weichen von diesen dagegen erheblich ab. Besonders in der Umgebung ihrer „Köpfe“ zeigen die Nullstellenverteilungsfunktionen des eindimensionalen Gases ein ganz absonderliches Verhalten.

Der vernünftige Verlauf der Nullstellenverteilungskurven der zwei- und dreidimensionalen Gasmodelle in Verbindung mit auch hinsichtlich der Kondensation wenigstens qualitativ befriedigenden Zustandsgleichungen scheint zum wiederholten Male die Güte dieser beiden Modelle zu bestätigen.

In gleicher Weise ist sicher auch der nicht zu erwartende, pathologische Verlauf der Nullstellenverteilungskurven des eindimensionalen Gasmodells verknüpft mit seiner unzureichenden Zustandsgleichung, die nicht einmal eine Phasenumwandlung zuläßt.

Herrn Prof. Dr. H. Hartmann danke ich für wohlwollende Unterstützung meiner Arbeit. 